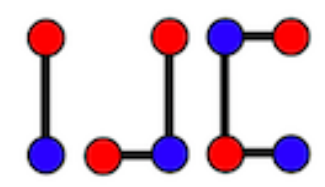

\title{
Structure of intersection graphs
}

\author{
Haval M. Mohammed Salih ${ }^{\mathrm{a}}$, Sanaa M. S. Omer ${ }^{\mathrm{b}}$ \\ ${ }^{a}$ Department of Mathematics, Faculty of Science, Soran University, Kawa St. Soran, Iraq \\ ${ }^{b}$ Department of Mathematics, Faculty of Science, University of Benghazi, Al Kufra, Libya \\ haval.mohammed@soran.edu.iq
}

\begin{abstract}
Let $G$ be a finite group and let $N$ be a fixed normal subgroup of $G$. In this paper, a new kind of graph on $G$, namely the intersection graph is defined and studied. We use $\Gamma_{G}^{i n}(N)$ to denote this graph, with its vertices are all normal subgroups of $G$ and two distinct vertices are adjacent if their intersection in $N$. We show some properties of this graph. For instance, the intersection graph is a simple connected with diameter at most two. Furthermore we give the graph structure of $\Gamma_{G}^{\text {in }}(\{e\})$ for some finite groups such as the symmetric, dihedral, special linear group, quaternion and cyclic groups.
\end{abstract}

Keywords: connected graph, normal subgroup, diameter

Mathematics Subject Classification: 20B40

DOI: 10.19184/ijc.2021.5.2.6

\section{Introduction}

Recently, study algebraic structures by graphs associated with them gives rise to many interesting results. Therefore, many mathematicians could associate the group theory with graph theory such as [1] and [4]. It has been proved that graphs can be interesting tools for the study of groups.

In the following context, some basics and related works are provided. Let $H$ be a group. A non empty subset $S$ of $H$ is called subgroup if $S$ is a group and denoted by $S \leq H$. A subgroup $S$ of $H$ is called normal if $h^{-1} s h \in S$ for all $h \in H$ and $s \in S$ [2]. A normal subgroup $N$ of $H$ is a minimal normal subgroup of $H$ if 1 and $N$ are the only normal subgroups of $H$ that are contained in $N$. A finite group is solvable if all its composition factors are cyclic of prime order.

Received: 20 June 2021， Revised: 5 December 2021 Accepted: 18 December 2021. 
Let $\mathbb{F}_{q}$ be a finite field. Then the general linear group $G L\left(n, \mathbb{F}_{q}\right)$ is the group of invertible $n$ by $n$ matrices with entries in $\mathbb{F}_{q}$ under matrix multiplication. We define the special linear group, $S L\left(n, \mathbb{F}_{q}\right)=\left\{A \in G L\left(n, \mathbb{F}_{q}\right):|A|=1\right\}$.

Theorem 1.1. [2] Let $N$ be a minimal normal subgroup of $H$. For all normal subgroups $M$ of $H$ either $N \leq M$ or $N \cap M=1$.

A graph is connected if there is a path connecting any two distinct vertices. The distance between two distinct vertices is the length of the shortest path connecting them (if such a path does not exist, define $\infty$. The diameter of a graph $G$, denoted by $\operatorname{diam}(G)$, is defined by the supremum of the distances between vertices. The girth of a graph, denoted $g(G)$ is the length of the shortest cycle in the graph $G$. A graph with no cycles has infinite girth. The minimum among all the maximum distances between a vertex to all other vertices is considered as the radius of the graph $G$ and denoted by $\operatorname{rad}(G)$.

The $r$-partite graph is one whose vertex can be partitioned into $r$ subsets so that an edge has both ends in no subset. A complete $r$-partite graph is an $r$-partite graph in which each vertex is adjacent to every vertex that is not in the same subset. The complete bipartite graph with part sizes $m$ and $n$ is denoted by $K_{m, n}$. A graph is called a complete if each pair of vertices is joined by an edge. We use $K_{n}$ to denote the complete graph with $n$ vertices. Two graphs $G$ and $H$ are isomorphic, denoted by $G \cong H$, if there is a bijection $\phi: G \rightarrow H$ of vertices such that the vertices $x$ and $y$ are adjacent in $G$ if and only if $\phi(x)$ and $\phi(y)$ are adjacent in $H$. A connected graph can be drawn without any edges crossing, it is called planar. A vertex $v$ of a connected graph $G$ is called a cut vertex of $G$, if $G \backslash v$ (Delete $v$ from $G$ ) results in a disconnected graph. Removing a cut vertex from a graph breaks it into two or more graphs. A connected graph with no cycles is called a tree. [3]. Throughout this paper we consider a finite simple un-directed graph.

Theorem 1.2 (Kuratowski's Theorem). [3] A graph is non-planar if and only if it contains a subgraph homeomorphic to $K_{3,3}$ or $K_{5}$.

\section{Some properties of the intersection graphs}

In this section, we study the intersection graph of finite groups $G$. The structure of $G$ is determined. As well, some related results are obtained.

Definition 2.1. Let $G$ be a finite group and $\mathcal{N}(G)$ be the set of all normal subgroups of $G$ and $N \in \mathcal{N}(G)$. The intersection graph denoted by $\Gamma_{G}^{i n}(N)$ is a undirected graph whose vertex set is $\mathcal{N}(G)$ and two distinct vertices are adjacent if their intersection lies in $N$.

Example 2.1. Consider the group of integer modulo 8, that is $\mathbb{Z}_{8}$. The interaction normal graphs for normal subgroups $\{0\},\{0,4\},\{0,2,4,6\}$ and $\mathbb{Z}_{8}$ are $\Gamma_{G}^{i n}(\{0\})=K_{1,3}, \Gamma_{G}^{i n}(\{0,4\})=K_{4} \backslash$ $\{$ one - edge $\}$ and $\Gamma_{G}^{\text {in }}(\{0,2,4,6\})=\Gamma_{G}^{\text {in }}\left(\mathbb{Z}_{8}\right)=K_{4}$.

Example 2.2. Consider the Klein four group $V_{4}=\{e, a, b, c\}$ where $a^{2}=b^{2}=c^{2}=e$ and $a b=b a, a c=c a, b c=c a$. The interaction graph $\Gamma_{G}^{i n}(\{e\})$ is given in Figure 1. 


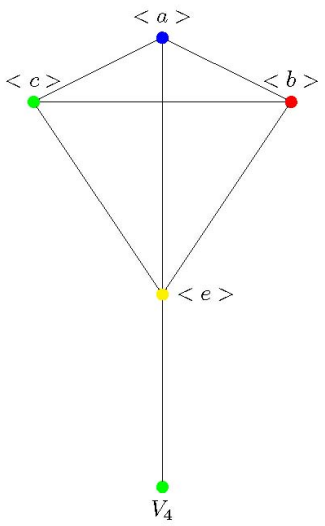

Figure 1. This is the caption of figure

Remark 2.1. 1. A finite group $G$ is simple if and only if $\Gamma_{G}^{i n}(N)=K_{2}$.

2. The number of edges of $\Gamma_{G}^{i n}(N)$ between $p-1$ and $\frac{p(p-1)}{2}$, where $|\mathcal{N}(G)|=p$.

Lemma 2.1. Let $N_{1}, N_{2}, \ldots, N_{l}$ be normal subgroups of a finite group $G$. Then

1. $\Gamma_{G}^{i n}(G)=K_{|\mathcal{N}(G)|}$.

2. $\Gamma_{G}^{i n}\left(\bigcap_{i \in \Lambda} N_{i}\right)=\bigcap_{i \in \Lambda} \Gamma_{G}^{i n}\left(N_{i}\right)$.

Proof. The proof is clear.

Lemma 2.2. Let $N$ be a minimal normal subgroup of $G$ and $L$ be a non trivial normal subroup of G. Then $\operatorname{deg}_{\Gamma_{G}^{i n}(L)}(\{e\})=\operatorname{deg}_{\Gamma_{G}^{i n}(L)}(N)=|\mathcal{N}(G)|$.

Proof. Based on Theorem 1.1, thus $N \cap L=N$ or $N \cap L=\{e\}$. In both cases we obtain $N \subseteq L$ or $\{e\} \subset L$. Thus $\operatorname{deg}_{\Gamma_{G}^{i n}(L)}(N)=|\mathcal{N}(G)|$.

Proposition 2.1. If $G$ has normal subgroups $N_{i}$ such that $\{e\}=N_{0} \subset N_{1} \subset N_{2} \subset \ldots \subset N_{r} \subset$ $N_{r+1}=G$, then

1. $\Gamma_{G}^{i n}(G)$ and $\Gamma_{G}^{i n}\left(N_{r}\right)$ are identical.

2. $\Gamma_{G}^{i n}(\{e\})=K_{1, r+1}$

3. $\Gamma_{G}^{i n}\left(N_{i}\right)$ is a subgraph of $\Gamma_{G}^{i n}\left(N_{l}\right)$ where $l>i$.

Proof. First, it is clear that they have the same number of vertices. Let $e=N_{i} N_{j}$ be an edge in $\Gamma_{G}^{i n}\left(N_{r}\right)$, that is $N_{i} \cap N_{j} \subseteq N_{r} \subseteq G$. This implies that $e=N_{i} N_{j}$ is an edge in $\Gamma_{G}^{i n}(G)$. On the other hand, let $e=N_{i} N_{j}$ be an edge in $\Gamma_{G}^{i n}(G)$, that is $N_{i} \cap N_{j} \subseteq G$. If either $N_{i}$ or $N_{j}$ is $N_{r}$, then we are done. If neither $N_{i}$ nor $N_{j}$ is $N_{r}$, then $N_{i} \cap N_{j}=N_{\bar{r}} \subseteq N_{r}$ where $\bar{r}=\min \{i, j\}$. Thus $e$ is an edge of $\Gamma_{G}^{i n}(G)$. Second, Since $N_{i} \cap N_{j}=N_{l} \not \subset\{e\}$ for $i, j \in\{1,2, \ldots, r+1\}$ where $l=\min \{i, j\}$ and $N_{i} \cap\{e\}=\{e\}$, then the result follows. Third, the proof is clear. 
As a direct consequence of Proposition 2.1, the following results are obtained.

Corollary 2.1. If $G$ has normal subgroups $N_{1}, \ldots, N_{r}$ such that $\{e\} \subseteq N_{1} \subseteq \ldots \subseteq N_{r} \subseteq G$.

1. If it has length 3, then $\Gamma_{G}^{i n}(\{e\}) \subseteq \Gamma_{G}^{i n}\left(N_{1}\right) \subseteq \Gamma_{G}^{i n}(G)$ or $\left(K_{1,2} \subseteq K_{3} \subseteq K_{3}\right)$.

2. If it has length 4, then $\Gamma_{G}^{i n}(\{e\}) \subseteq \Gamma_{G}^{i n}\left(N_{1}\right) \subseteq \Gamma_{G}^{i n}\left(N_{2}\right) \subseteq \Gamma_{G}^{i n}(G)$ or $\left(K_{1,3} \subseteq K_{4} \backslash\{\right.$ one edge $\left.\} \subseteq K_{4} \subseteq K_{4}\right)$.

Proposition 2.2. If $G$ is a finite solvable group and $\Gamma_{G}^{i n}(\{e\})=K_{1,2}$, then

1. $G$ is a cyclic p-group of order $p^{2}$.

2. $G$ is a semidirect product $G=P \rtimes Q$, where $P$ is an elementary abelian p-group and $Q$ is a cyclic group of order $q$, with $p$ and $q$ being distinct primes.

Proof. Since $\Gamma_{G}^{i n}(\{e\})=K_{1,2}$, then $G$ has a unique non trivial normal subgroup. The proof of rests follow from Theorem 1.2 in [5].

Note that the converse of 1 . in Proposition 2.2 is true and it can be seen in Proposition 3.3. The following example shows that the converse of 2. in Proposition 2.2 is not true.

Example 2.3. The group $A G a m m a L(1,8)$ is the semi-direct product of $C_{8}$ by $C_{7}: C_{3}$. But $\Gamma_{G}^{i n}(\{e\}) \neq K_{1,3}$.

Proposition 2.3. If $G$ is a finite non solvable group and $\Gamma_{G}^{i n}(\{e\})=K_{1,2}$, then $G$ describe in Theorem 1.2 in [5].

Proof. The proof is similar as Proposition 2.2.

Example 2.4. Consider the group $G=G L(2,3)$ and it has 5 normal subgroups such as $\{e\}, C_{2}, Q_{8}$, $S L(2,3)$ and $G L(2,3)$. The corresponding intersection graphs are $K_{1,4} \subseteq H \subseteq K_{4} \backslash\{e\} \subseteq K_{4} \subseteq$ $K_{4}$ where the intersection graph of $H$ is given in Figure 2. 


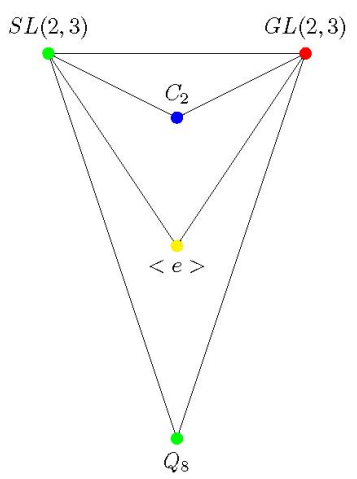

Figure 2. The intersection graph of $H$

Lemma 2.3. Let $\Gamma_{G}^{i n}(N)$ be the intersection graph. Then

$$
d\left(H_{i}, H_{j}\right)= \begin{cases}1 & \text { if } H_{i} \cap H_{j} \subseteq N \\ 2 & \text { if } H_{i} \cap H_{j} \nsubseteq \subseteq N\end{cases}
$$

Proof. The proof is clear.

Proposition 2.4. Let $G$ be a finite group and $N$ be a normal subgroup of $G$. Then $\Gamma_{G}^{i n}(N)$ is connected graph with diameter at most 2 and radius 1.

Proof. The proof is clear.

Proposition 2.5. Let $G$ be a finite group with at least two minimal normal subgroups. Then $\Gamma_{G}^{i n}(\{e\})$ is not tree graph. Furthermore, $\Gamma_{G}^{i n}(N)$ has girth 3.

Proof. Since $G$ has at least two minimal normal subgroups $N_{1}$ and $N_{2}$, thus the normal subgroups with trivial normal subgroup gives the cycle $K_{3}$ in $\Gamma_{G}^{i n}(\{e\})$. Therefore $\Gamma_{G}^{i n}(\{e\})$ is not tree. Based on Proposition 2.1, $\Gamma_{G}^{i n}(\{e\})$ is a subgraph of $\Gamma_{G}^{i n}(N)$. Hence $\Gamma_{G}^{i n}(N)$ has girth 3.

Theorem 2.1. Let $G$ be a finite group with non trivial normal proper subgroups $N_{1}, \ldots, N_{r}$. If $\left|E\left(\Gamma_{G}^{i n}\left(N_{i}\right)\right)\right|=\left|E\left(\Gamma_{G}^{i n}\left(N_{j}\right)\right)\right|$ and $\left|N_{i}\right|=\left|N_{j}\right|$ for some $i, j$. Then $\Gamma_{G}^{i n}\left(N_{i}\right)$ and $\Gamma_{G}^{i n}\left(N_{j}\right)$ are isomorphic.

Proof. Define $f: V\left(\Gamma_{G}^{i n}\left(N_{i}\right)\right) \rightarrow V\left(\Gamma_{G}^{i n}\left(N_{j}\right)\right)$ by $f\left(H_{i}\right)=H_{j}, f\left(H_{j}\right)=H_{i}$ and $f\left(H_{l}\right)=H_{l}$ where $l \neq i, j$. It is clear that $f$ is bijective. Let $H_{m} H_{s}$ be an edge in $\Gamma_{G}^{i n}\left(N_{i}\right)$ that is $H_{m} \cap H_{s} \subseteq H_{i}$. If $m, s \neq i, j$ then $f\left(H_{m} \cap H_{s}\right) \subseteq f\left(H_{i}\right)$, that is $H_{m} \cap H_{s} \subseteq H_{j}$. Thus, $H_{m} H_{s}$ is an edge in $\Gamma_{G}^{i n}\left(N_{j}\right)$. If $m=i$ or $s=i(m=j$ or $s=j)$, then the result follows.

The following examples show that the converse of Theorem 2.1 is not true. 
Example 2.5. Let $G=C_{21}$ be a cyclic group. Then, $\Gamma_{G}^{i n}\left(C_{3}\right) \cong \Gamma_{G}^{i n}\left(C_{7}\right)$.

Example 2.6. The groups $G L(3,3)$ and $G L(3,8)$ have the same number of normal subgroups. Thus, their intersection graphs are identical.

Proposition 2.6. Let $G$ be a finite group with at least four minimal normal subgroups. Then $\Gamma_{G}^{\text {in }}(N)$ is not planar graph.

Proof. Suppose that $G$ is finite group with four minimal normal subgroups $N_{i}$ for $i=1,2,3,4$. It is clear that $N_{i} \cap N_{j}=\{e\}$ for $i \neq j$. These minimal normal subgroups together with trivial normal group produce $K_{5}$ in $\Gamma_{G}^{i n}(\{e\})$. From Theorem 1.2, the result is obtain.

Proposition 2.7. Let $\Gamma_{G}^{i n}(\{e\})$ be the intersection graph, with at least two minimal normal subgroups of $G$ then $\{e\}$ is a cut vertex.

Proof. If $\Gamma_{G}^{i n}(\{e\})$ is a star graph, then the proof is clear. If $\Gamma_{G}^{i n}(\{e\})$ is not star graph, then using Lemma 2.2, $\operatorname{deg}_{\Gamma_{G}^{i n}(\{e\})}(\{e\})=l$ where $l=|\mathcal{N}(G)|$ and $\operatorname{deg}_{\Gamma_{G}^{i n}(\{e\})}(G)=1$. Therefore, vertex $G$ is isolated vertex in $\Gamma_{G}^{i n}(\{e\}) \backslash\{e\}$. Thus, the graph is disconnected and $\{e\}$ is a cut vertex.

\section{The intersection graph of some special finite groups}

In this section, we find the intersection graph for the famous known groups.

Lemma 3.1. If $G$ is the symmetric group $S_{n}$ and $n>2$, then

$$
\Gamma_{G}^{i n}(\{e\})=\left\{\begin{array}{l}
K_{1,3} \text { if } n=4, \\
K_{1,2} \text { otherwise }
\end{array}\right.
$$

Proof 3.1. It is known that $S_{4}$ has four normal subgroups as follows: $\{e\} \subseteq C_{2} \subseteq C_{2} \times C_{2} \subseteq$ $A_{4} \subseteq S_{4}$ and otherwise we have $\{e\} \subseteq A_{n} \subseteq S_{n}$. By Proposition 2.1, the result follows.

Lemma 3.2. If $G=S L(3, q)$ where $q$ is a prime power, then

$$
\Gamma_{G}^{i n}(\{e\})=\left\{\begin{array}{l}
K_{1,2} \text { if } q \cong 1 \bmod 3 \\
K_{2} \text { otherwise }
\end{array}\right.
$$

Proof. The proof is clear.

Lemma 3.3. If $G=S L(4, q)$ where $q$ is a prime power, then

$$
\Gamma_{G}^{i n}(\{e\})=\left\{\begin{array}{l}
K_{1,3} \text { if } q \cong 1 \quad \bmod 4, \\
K_{2} \text { if } q \cong 0 \quad \bmod 4, \\
K_{1,2} \text { if } q \cong 2 \bmod 4
\end{array}\right.
$$

Proof. The proof is clear. 
Proposition 3.1. Let $G \cong\left\langle a, b \mid a^{n}=e=b^{2}, b a b^{-1}=a^{-1}\right\rangle$ be the dihedral group $D_{2 n}$. Then

$$
\Gamma_{G}^{i n}(\{e\})=\left\{\begin{array}{l}
K_{1, \alpha+1}, \text { if } n \text { is odd, and } n=p^{\alpha}, \\
K_{1, \alpha+3}, \text { if } n \text { is even, and } n=p^{\alpha}, \\
\text { is not tree, if there exist distinct primes } p_{i} \text { and } p_{j} \\
\text { such that } n=p_{1}^{\alpha_{1} \ldots p_{r}^{\alpha_{r}} .} .
\end{array}\right.
$$

Proof. When $n$ is odd, it is clear that $G$ has $\alpha+2$ normal subgroups. The rest follows from Proposition 2.1. In the case that $n$ is even, thus $G$ has $\alpha+4$ normal subgroups. Recall Proposition 2.1 , then $K_{1, \alpha+3}$. Since $n=p_{1}^{\alpha_{1}} \ldots p_{r}^{\alpha_{r}}$, then without loss of generality we assume that $n=p_{1}^{\alpha_{1}} p_{2}^{\alpha_{2}}$. The proof of the rest follows from Proposition 2.5.

Proposition 3.2. Let $G=Q_{4 n}$ be a quaternion group. Then

$$
\Gamma_{G}^{i n}(\{e\})=\left\{\begin{array}{l}
K_{1, \alpha+4}, \text { if } n=2^{\alpha}, \\
\text { is not tree, } \text { otherwise }
\end{array}\right.
$$

Proof. The proof is similar as Proposition 3.1.

Proposition 3.3. If $G$ is a cyclic group of order $n=p_{1}^{\alpha_{1}} \ldots p_{k}^{\alpha_{k}}$ where $p_{i}$ are prime numbers, then

$$
\Gamma_{G}^{i n}(\{e\})=\left\{\begin{array}{l}
K_{1, \alpha_{1}}, \text { if } k=1, \\
\text { is not tree, otherwise }
\end{array}\right.
$$

Proof. If $k=1$ and $\alpha_{1}=1$ then $G$ is simple group. From Remark 2.1, we have $\Gamma_{G}^{i n}(\{e\})=K_{1,1}$ and the proof of $\alpha_{1}>1$ follows from Proposition 2.1. If $k \neq 1$, then without loss of generality we may assume that $n=p_{1}^{\alpha_{1}} p_{2}^{\alpha_{2}}$. So $G$ contains two minimal normal subgroups. These minimal normal subgroups with $\{e\}$ gives the cycle $C_{3}$. Therefore $\Gamma_{G}^{i n}(\{e\})$ is not tree graph.

Example 3.1. Consider the cyclic group $\mathbb{Z}_{m}$, where $m=2^{n} 3$, the graph $\Gamma_{G}^{i n}(\{e\})$ contains $1,2,3$ triangles if $n=1,2,3$ respectively.

\section{Conclusion}

In this study, we introduced a new graph called the intersection graph. The graph is found for the symmetric groups, special linear groups, dihedral groups and others. Furthermore, some properties of this graph were determined. We conclude this section with the following questions:

Question. 1. If $G$ is a cyclic group of order $n$. How many triangles are in $\Gamma_{G}^{\text {in }}(\{e\})$ ?

2. If $G=S L(5, q)$ and $q \cong 1 \bmod 5$. What is the structure of the graph $\Gamma_{G}^{i n}(\{e\})$ ?

3. If we replace vertex set with subgroups of $G$. What is the structure of the graph $\Gamma_{G}^{i n}(\{e\})$ ? 


\section{Acknowledgement}

The authors would like thanks to the referees for the reading, comments and suggestions which improved the paper.

\section{References}

[1] H.P. Tong-Viet, Finite groups whose prime graphs are regular Journal of Algebra 397, (2014), $18-31$.

[2] H. Kurzweil and B. Stellmacher, The theory of finite groups: an introduction, Springer Science \& Business Media, (2006).

[3] R.J. Wilson, Introduction to graph theory, Pearson Education India, (1979).

[4] A. Erfanian and B. Tolue, Conjugate graphs of finite groups, Discrete Mathematics, Algorithms and Applications 4(2) (2012), 1250035. https://doi.org/10.1142/S1793830912500358

[5] Z.Q. Hai and C.J. Ji, Finite groups whose nontrivial normal subgroups have the same order, Journal of Mathematical Research and Exposition 28(4) (2008), 807-812. 